\title{
Seven steps to diagnose delta-aminolevulinic acid deficiency porphyria
}

\author{
Adriana Grigore', Vlad Tiu', Elena Terecoasa ${ }^{1,2}$, Lavinia Tatu', \\ Ana Cobzaru', Cristina Tiu ${ }^{1,2}$ \\ ${ }^{1}$ Department of Neurology, University Emergency Hospital, Bucharest, Romania \\ 2"Carol Davila" University of Medicine and Pharmacy, Bucharest, Romania
}

\begin{abstract}
Delta-aminolevulinic acid dehydratase porphyria (ADP) is a rare cause of acute polyneuropathy. It should be part of the differential diagnosis in every patient presenting with polyneuropathy and elevated liver enzymes. Genetic, acquired and environmental factors can play a role in the clinical manifestations and there are several steps in reaching the diagnosis. We present the case of a male patient with at least two possible causes for his symptoms, the diagnostic process and the outcome.
\end{abstract}

Keywords: acute polyneuropathy, delta-aminolevulinic acid dehydratase porphyria, lead poisoning

\section{INTRODUCTION}

Acute polyneuropathies are usually severe disorders which require a rapid therapeutic intervention. A successful treatment and a good prognosis of the patient are obviously dependent on a correct diagnosis. Porphyrias represent a group of metabolic disorders (inherited or acquired) that result from the disruption of the heme biosynthetic pathway due to a deficiency of a specific enzyme. The most frequent is acute intermittent porphyria, but similar clinical aspect can be encountered in a more rare form, ADP, which is an acute hepatic porphyria that results from severe delta-aminolevulinic acid dehydratase (ALAD) deficiency.

\section{CLINICAL CASE}

A 32-year old, working in the IT field, was referred to our Department of Neurology for a recently installed quadriparesis, in December 2015.

His medical history was non-relevant, excepting two tonic-clonic seizures with loss of conscious- ness, one in 2013, not treated and the second in March 2015. The investigations undertaken in order to identify a cause for those seizures (brain magnetic resonance imaging and electroencephalography) were normal. In May 2015 he was hospitalised in an Internal Medicine Department for abdominal pain and diarrhoea. Routine blood analysis showed mildly increased aminotransferases, an elevated direct bilirubin level and a mild lymphopenia. He tested negative for viral hepatitis. Systemic lupus erythematosus and hemochromatosis were ruled out. He was started on Levetiracetam for his seizures.

A month before coming to our Department, he was admitted in another hospital for abdominal pain, nausea, vomiting, diarrhoea and progressive weight loss during the previous three weeks. He had elevated liver enzymes (over 5 times the upper normal limit) and high indirect bilirubin (3.5 mg/ $\mathrm{dl}$ ), but also severe anaemia (haemoglobin $5.5 \mathrm{~g} /$ dl). Subsequently, he developed ascending paresthesias and motor weakness involving all four 
limbs, with diminished deep tendon reflexes. The upper and lower gastrointestinal endoscopy and the abdominal computed tomography were normal. A lumbar puncture was performed, and cerebral spinal fluid analysis was unremarkable, and the patient was considered to have a Guillain-Barré syndrome (GBS) due to the acute ascending motor deficit.

He has afterwards referred to our Department, for intravenous immunoglobulin $G$ treatment. Upon admission, he had tetraparesis, more prominent proximal than distal, generalized hypotonia, muscle atrophies, mostly in the girdles, and absent deep tendon reflexes; he was unable to walk or to stand up without help, he could not perform the abduction of the arms, while the hands had a certain motility preserved (Fig. 1a, Fig. 1b).

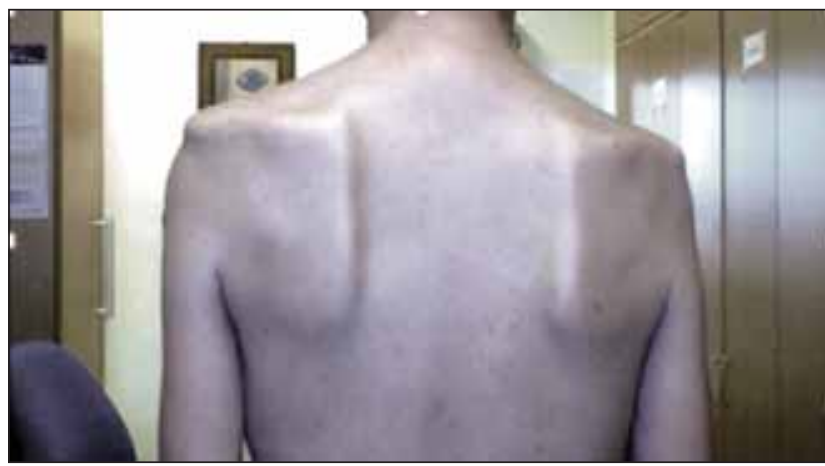

FIGURE 1A. Amyotrophies of supraspinatus, infraspinatus and deltoid muscles.

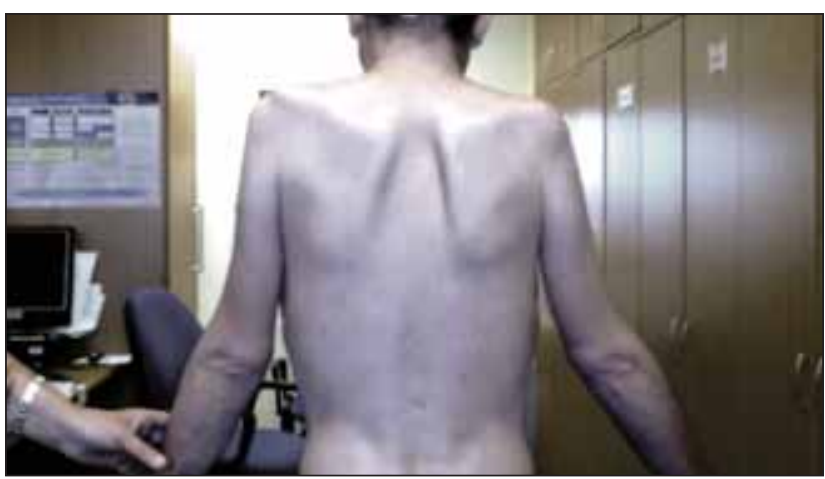

FIGURE 1B. Maximal range of movement at the attempt to abduct the upper limbs.

Blood analysis revealed slight hypochromic anaemia (haemoglobin $10 \mathrm{~g} / \mathrm{dL}$ ) and mildly elevated liver enzymes. Motor conduction studies revealed low CMAP (compound muscle action potential) amplitude in all examined nerves, with a slightly asymmetry for the peroneal nerves. The area of the CMAP and the conduction velocity were within normal limits and the latency in the right median nerve was a little increased. Sensory con- duction studies showed SNAP (sensory nerve action potential) amplitude in the lower limit of normal for the right peroneal nerve and right median nerve, with decreased velocity conduction for the latter. To sum up, the neuro-electrophysiological exam was suggestive of subacute severe predominantly motor axonal polyneuropathy (Fig. 2). A screening for vasculitis and infections (HIV, hepatitis B and C, syphilis) was negative.

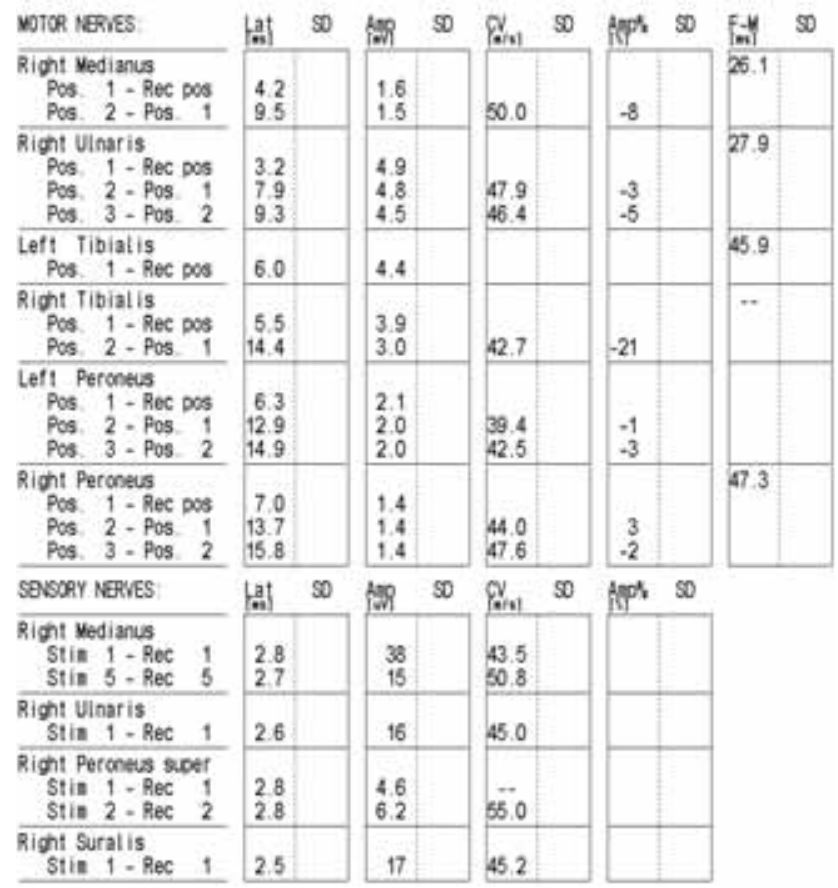

FIGURE 2. Results of the motor and sensory NCSs (nerve conduction studies).

Taking all the above into consideration, we performed blood and urine tests for porphyria; the results are detailed in Table 1. They were consistent with delta-aminolevulinic acid dehydratase porphyria.

TABLE 1. Laboratory results.

\begin{tabular}{|l|l|c|c|}
\hline \multicolumn{2}{|l|}{ Laboratory test } & Result & Normal range \\
\hline \multirow{4}{*}{$\begin{array}{l}\text { 24-hour } \\
\text { urine }\end{array}$} & Porphobilinogen & $2.28 \mathrm{mg}$ & $1.9 \mathrm{mg}$ \\
\cline { 2 - 4 } & Total porphyrins & $1051.03 \mu \mathrm{g}$ & $<220 \mu \mathrm{g}$ \\
\cline { 2 - 4 } & Uroporphyrins & $106.65 \mu \mathrm{g}$ & $15-50 \mu \mathrm{g}$ \\
\cline { 2 - 4 } & Coproporphyrins & $944.38 \mu \mathrm{g}$ & $35-150 \mu \mathrm{g}$ \\
\cline { 2 - 4 } & Delta-aminolevulinic acid & $30.75 \mathrm{mg}$ & $<6 \mathrm{mg}$ \\
\hline Blood & ALAD & $0.5 \mathrm{U} / \mathrm{L}$ & $>14.5 \mathrm{U} / \mathrm{L}$ \\
\hline
\end{tabular}

During his stay in our Department, the patient received intravenous glucose and underwent physical therapy, which he continued after discharge. He returned for a follow-up two years later, being able to walk unassisted and with mild quadripare- 
sis. A repeated NCSs also showed slightly improvement in both motor conduction velocities and amplitudes. We repeated the serum ALAD testing that showed a persistent low level.

\section{DISCUSSION}

The case is offering an opportunity to review the algorithm for diagnosing a motor deficit involving all four limbs, with an acute onset and rapid progression.

1. Facing such a patient the clinician has to decide if the symptoms belong to an upper or lower motor neuron syndrome. Although this is easy to do in most of the cases, the preservation of deep tendon reflexes or a certain level of sensory deficit associated to the lower motor neuron deficit can sometimes mislead toward a central nervous system involvement. Our patient had obviously a lower motor neuron involvement, with deep tendon reflexes diminished, no sensory involvement beside mild paresthesias.

2. The onset was acute (evolving over a period of days up to one month), with rapid progression, involving all four limbs. The distribution was symmetrical, but the limbs were affected proximal more than distal. Among the acute polyneuropathies, GBS is the most frequent, but usually the classic Landry form begins distally, in the inferior limbs, and has an ascending course. Other con `s for GBS were the lack of pain and of proprioceptive sensory involvement, the absence of respiratory disturbances or of dysautonomic signs and symptoms despite the severity of the motor deficit. Because there are also exceptions from this classic aspect, and the motor deficit can start proximally in the inferior limbs, or the disease can begin in the superior limbs, the clinical aspect is not enough in order to differentiate GBS from other acute polyneuropathies. The CSF exam was not contributory, since the aspect was completely normal; CSF can be normal in the first week and usually gets the typical albuminocytologic dissociation aspect by the second week. The spectrum of acute polyneuropathies is very large, including acute episodes of chronic, demyelinating polyneuropathies, diphtheria, vitamin B12 deficit, Lyme disease, neuropathy of intensive care, different toxic substances, vasculitis or metabolic disorders such as porphyrias.
Some of them are easy to exclude by the lack of medical history or of clinical context, but for the others we need further investigation.

3. The next mandatory step is the neuro-electrophysiological examination (EMG), in order to differentiate between a demyelinating and an axonal form of polyneuropathy. Our patient had a subacute (EMG was performed at almost 2 months from the onset), predominantly motor axonal polyneuropathy, superior limbs being more affected. Although axonal forms are described in GBS syndrome, most of cases are demyelinating. The aspect of the EMG, combined with the other clinical signs beside the motor deficit (seizures, abdominal pain, diarrhoea) and lab findings (anaemia, elevated aminotransferases) pointed toward porphyria.

4. Porphyrias are a group of metabolic disorders, mostly inherited, characterized by different enzymatic defects along the pathway of heme biosynthesis. The lack of one enzyme leads to the accumulation of a certain heme precursor, either in the erythrocytes, or in the liver. Erythropoietic porphyrias have mainly dermatological manifestations, while the hepatic porphyrias lead to neurological signs and symptoms, with the exception of porphyria cutanea tarda. From the list of hepatic porphyrias leading to neurological dysfunction, hereditary coproporphyria and porphyria variegate also have cutaneous lesions, which were absent in our patient. This leaves us in the situation of searching for either acute intermittent porphyria, or for an ALAD deficit. The central nervous system involvement is characterized by seizures and psychiatric manifestations (mainly behavioural changes, with agitation). The peripheral neuropathy is the last to appear (after the digestive and central nervous system signs); it is mainly motor, affecting the upper limbs more proximal than distal, reaching its nadir one month after the onset of the attack. It is determined by the oxidative stress induced by the accumulation of heme precursors combined with the energy failure due to the reduced heme production. ${ }^{1}$ Clinical manifestations do not differ between these two types of porphyria, and the differential diagnosis is based entirely on lab findings.

5. ALAD, also called porphobilinogen synthase, is involved in the second step of the heme synthesis pathway, forming porphobilinogen from two molecules of delta-aminolevulinic acid, but the most fre- 
quent form is acute intermittent porphyria, caused by the deficiency of porphobilinogen deaminase (Fig. 3).

If we take a look to the steps of the heme synthesis pathway, we see that starting with acute intermittent porphyria, all the types of porphyria should have both delta-aminolevulinic acid (ALA) and porphobilinogen (PBG) level increased, while in ALAD porphyria, the level of PBG is normal and only ALA is increased. In our patient urinary PBG was $2.28 \mathrm{mg} / 24 \mathrm{~h}$, very slightly increased com-

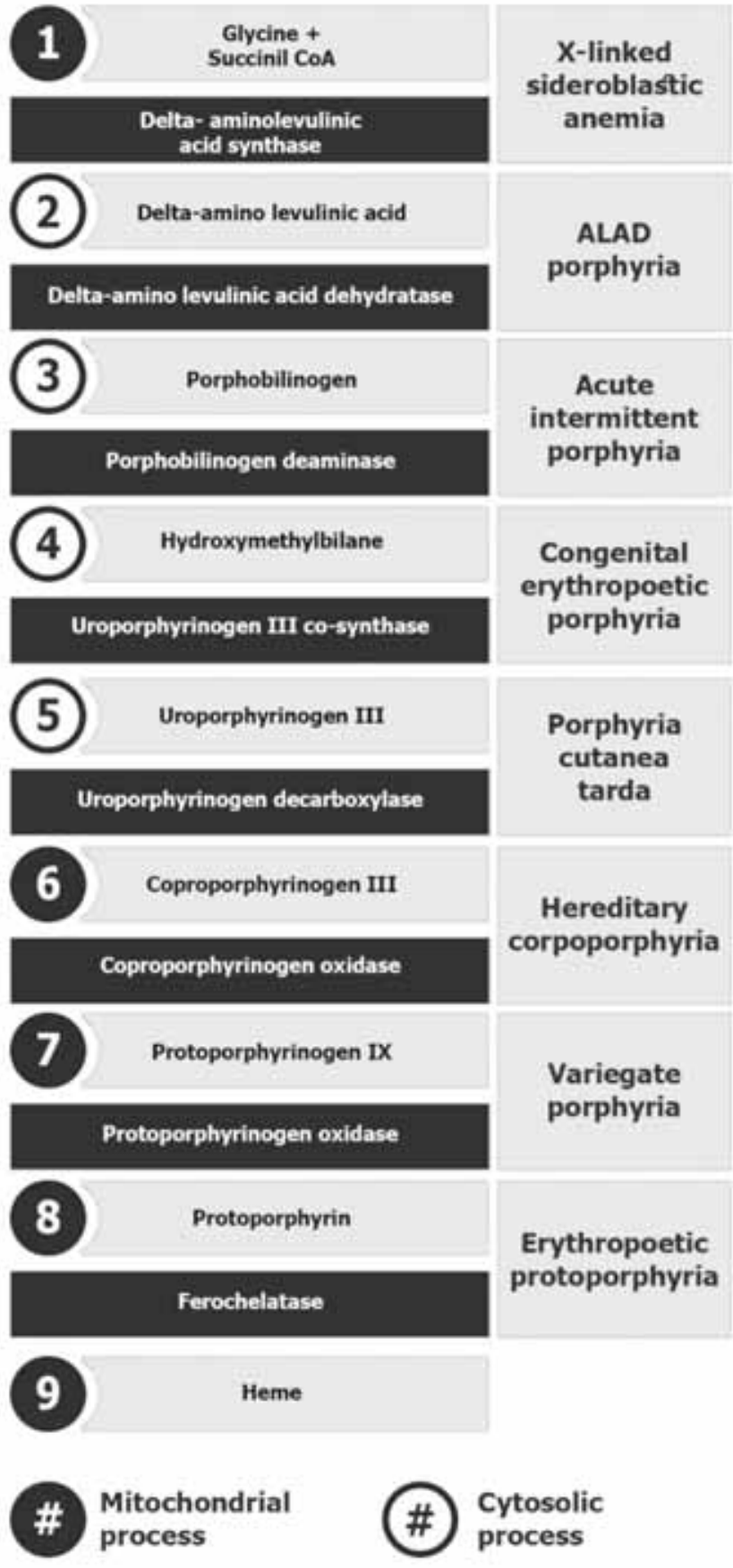

FIGURE 3. Heme synthesis pathway. pared to the normal upper limit of $1.9 \mathrm{mg} / 24 \mathrm{~h}$, while ALA level was fivefold increased, and this determined us to rule out acute intermittent porphyria and incline towards the rarer ALAD porphyria. We performed the serum dosage of ALA dehydratase, and the deficit was confirmed.

6. The last stage of the diagnostic work-up was to identify the cause of ALAD deficiency.

ALAD deficiency may be caused by an autosomal recessive disorder (ADP) or by a variety of factors, such as lead poisoning, hereditary tyrosinemia type 1 , exposure to iron, trichloroethylene or styrene; furthermore, it can also be associated with more frequent conditions such as diabetes mellitus, hemodialysis for chronic kidney disease, drugs that are metabolized via cytochrome P 450, smoking and alcoholism (2-5).

ADP is the rarest form of inherited porphyrias. The concept of ADP was first introduced by Doss et al. in 1979, and since then only six cases have been confirmed on a molecular level worldwide, all of them being males (6-12). Eleven ALAD gene mutations have been identified until the present moment (12-14). Five out of the six patients had compound heterozygous mutations and were under the age of 20 at the onset of symptoms; the sixth patient had one heterozygous mutation, but also had a red cell line myeloproliferative disorder, which decreased the erythrocyte production of ALAD (6,912).

Clinical manifestations depend on the level of ALAD $(15,16)$. There is a physiologic excess of ALAD in contrast to all the other enzymes involved in the heme synthesis, therefore taking into consideration the data reported in the literature so far, it appears that in order for a patient to have the specific symptoms, there has to be one of three scenarios: compound heterozygous mutations; one heterozygous mutation with a precipitating factor; or, a severe enough external factor (such as lead poisoning or alcohol intake) (10,17-19).

Since the genetic defect is so rare and difficult to prove, we looked for external factors able to precipitate an acute attack of porphyria and determine the acute polyneuropathy. At the beginning the patient and his family strongly denied alcohol consumption and there was no history of concomitant medication with drugs metabolized via Cytochrome P 450; therefore, we looked for a possible lead poi- 
soning. The results were striking, with very high levels of lead in serum and urine (Table 2).

TABLE 2. Lead levels in serum and urine.

\begin{tabular}{|l|c|c|}
\hline Laboratory test & Result & Normal range \\
\hline $\mathbf{2 4} \mathrm{h}$ urine Lead & $354.75 \mu \mathrm{g}$ & $\mathrm{N}<8 \mu \mathrm{g}$ \\
\hline Serum Lead & $40.53 \mu \mathrm{g} / \mathrm{dl}$ & $\mathrm{N}<25 \mu \mathrm{g} / \mathrm{dl}$ \\
\hline
\end{tabular}

7. Where did the lead come from? The patient finally admitted that he was a heavy drinker and that he drank mostly strong alcohol, manufactured in the countryside in a crafted boiler with lead jointing. We asked the family to take samples of the alcohol and of the fountain water from the countryside home (where the patient spend most of the time) and we found out that the lead level was $105.55 \mu \mathrm{g} / \mathrm{l}$ in the alcohol, and $8.45 \mu \mathrm{g} / \mathrm{l}$ in the fountain water, much above the admitted limits.

\section{CONCLUSION}

We concluded that the underlying cause of the porphyria was a chronic exposure to lead and chronic alcohol ingestion. However, we cannot rule out a possible single heterozygous mutation of the ALAD gene, as the serum enzyme level was persistently decreased two years after diagnosis, without apparent exposure to lead and with no reported alcohol intake.

Conflict of interest: none declared Financial support: none declared

\section{REFERENCES}

1. Lin CS-Y, Park S.B., Krishnan A.V. Porphyric neuropathy. In: Handbook of clinical neurology. 2013. p. 613-27.

2. Sassa S. ALAD Porphyria. Semin Liver Dis 1998;18(1):95-101.

3. Sieg I., Doss M.O., Kandels H., Schneider J. Effect of alcohol on delta-aminolevulinic acid dehydratase and porphyrin metabolism in man. Clin Chim Acta 1991; 202(3):211-8.

4. Pagliuca A., Mufti G.J., Baldwin D., Lestas A.N., Wallis R.M., Bellingham A.J. Lead poisoning: clinical, biochemical, and haematological aspects of a recent outbreak. J Clin Pathol 1990; 43(4):277-81.

5. Mitchell G., Larochelle J., Lambert M. et al. Neurologic Crises in Hereditary Tyrosinemia. N Engl J Med 1990; 322(7):432-7.

6. Doss M., von Tiepermann R., Schneider J., Schmid H. New type of hepatic porphyria with porphobilinogen synthase defect and intermittent acute clinical manifestation. Klin Wochenschr 1979; 57(20):1123-7.

7. Akagi R., Shimizu R., Furuyama K., Doss M.O., Sassa S. Novel molecular defects of the delta-aminolevulinate dehydratase gene in a patient with inherited acute hepatic porphyria. Hepatology 2000; 31(3):704-8.

8. Ishida N., Fujita H., Fukuda Y. et al. Cloning and expression of the defective genes from a patient with delta-aminolevulinate dehydratase porphyria. J Clin Invest 1992; 89(5):1431-7.

9. Thunell S., Holmberg L., Lundgren J. Aminolaevulinate dehydratase porphyria in infancy. A clinical and biochemical study. J Clin Chem Clin Biochem 1987; 25(1):5-14.

10. Hassoun A., Verstraeten L., Mercelis R., Martin J.J. Biochemical diagnosis of an hereditary aminolaevulinate dehydratase deficiency in a 63-year-old man. J Clin Chem Clin Biochem 1989; 27(10):781-6.
11. Doss M.O., Stauch T., Gross U. et al. The third case of Doss porphyria (-amino-levulinic acid dehydratase deficiency) in Germany. J Inherit Metab Dis 2004; 27(4):529-36.

12. Akagi R., Kato N., Inoue R., Anderson K.E., Jaffe E.K., Sassa S. delta-Aminolevulinate dehydratase (ALAD) porphyria: the first case in North America with two novel ALAD mutations. Mol Genet Metab 2006; 87(4):329-36.

13. Maruno M., Furuyama K., Akagi R. et al. Highly heterogeneous nature of delta-aminolevulinate dehydratase (ALAD) deficiencies in ALAD porphyria. Blood 2001; 97(10):2972-8.

14. Jaffe E.K., Stith L. ALAD porphyria is a conformational disease. Am J Hum Genet 2007; 80(2):329-37.

15. Akagi R., Yasui Y., Harper P., Sassa S. A novel mutation of delta-aminolaevulinate dehydratase in a healthy child with $12 \%$ erythrocyte enzyme activity. Br J Haematol 1999; 106(4):931-7.

16. Bird T.D., Hamernyik P., Nutter J.Y., Labbe R.F. Inherited deficiency of delta-aminolevulinic acid dehydratase. Am J Hum Genet 1979; 31(6):662-8.

17. Jaffe E.K., Martins J., Li J., Kervinen J., Dunbrack R.L. The molecular mechanism of lead inhibition of human porphobilinogen synthase. J Biol Chem 2001; 276(2):1531-7.

18. Bergdahl I.A., Grubb A., Schütz A. et al. Lead binding to Pharmacol Toxicol 1997; 81(4):153-8.

19. Doss M., Laubenthal F., Stoeppler M. Lead poisoning in inherited delta-aminolevulinic acid dehydratase deficiency. Int Arch Occup Environ Health 1984; 54(1):55-63. delta-aminolevulinic acid dehydratase (ALAD) in human erythrocytes. 